\title{
A Survey of Atmospheric Pollen and Spores in North-Western Nigeria
}

\author{
Olusola H. ADEKANMBI*, Olugbenga S. ALEBIOSU \\ University of Lagos, Faculty of Science, Department of Botany, Laboratory of Palynology and Palaeobotany, Akoka, Yaba, Lagos, \\ Nigeria; helen_olu@yahoo.com (*correspondingauthor);olugbengaalebiosu@gmail.com
}

\begin{abstract}
The incidence of airborne pollen occurring as aeroallergens of varied levels at different periods of the year has generated some public health concern globally. The present study was conducted in order to examine the monthly depositional rate of airborne pollen and fern spores, as well as the impact of meteorological parameters and local plants on pollen distribution at two study sites in Kaduna and Sokoto States of North Western Nigeria. The study spanned a period of one year, during which a modified Tauber pollen sampler was employed in the collection of atmospheric pollen and fern spores; local plants were also enumerated. Residual solution was collected monthly, subjected to acetolysis and the treated residues were viewed for microscopic studies. The results reveal monthly distribution of airborne pollen and fern spore types at the study sites during the sampling period. Dominant pollen types in Kaduna State were those of Alchornea laxiflora, Pinus caribaea, Terminalia sp., Sapotaceae and Poaceae family. Pollen of Syzygium guineense, Alchornea sp., Sapotaceae and Poaceae dominated in Sokoto State. Spores of Pteris sp., Nephrolepis sp. and a trilete fern were also recovered at both locations. Some local plants in the immediate vegetation contributed to the aeroflora of these locations. No significant correlation was recorded between monthly total pollen concentrations and mean monthly meteorological parameters of the locations. It is imperative to investigate the allergenicity of these dominant pollen types, so as to aid pollen hypersensitive individuals in adopting appropriate prophylactic measures.
\end{abstract}

Keywords: airborne; allergenicity; dominant; local plants; meteorological

\section{Introduction}

The study of pollen grains, fungal spores, insect debris, dust mites and organic dust, dispersed into the atmosphere as particles of biological origin is referred to as aerobiology (Singh and Kumar, 2004). Information about the type and abundance of airborne pollen grains at a given time in an area could be obtained through the concept of aeropalynology. The allergen proteins of pollen are immunomodulatory substances, playing a crucial role in the sensitization and/or exacerbation of these allergies (D'Amato et al., 2002; Kamijo et al., 2013). The common symptoms of allergy hypersensitivity are bronchial asthma, atopic dermatitis, rhinoconjunctivitis and a general feeling of malaise, among others (Singh and Mathur, 2012).

In Nigeria, some aeropalynological work have been recently conducted in various parts of the country and these include investigating dominant pollen and fern spore types (Adekanmbi and Ogundipe, 2010; Adeonipekun, 2012; Abdulrahman et al., 2015; Ajikah et al., 2015, 2017; Adekanmbi et al., 2017), examining correlations between pollen data and meteorological parameters (Ezike et al., 2016; Ajikah et al., 2017; Alebiosu et al., 2017); relationships between airborne pollen load and medical data (Adeniyi et al., 2014); inferences on their health risk periods (Adeniyi et al., 2014; Ezike et al., 2016; Alebiosu et al., 2017).

Essien and Aina (2014) quoted Singh and Rawat (2000), stating that most pollen grains found as allergens are wind-pollinated, characteristic of a light-weight and may be dispersed to considerable distances. They further cited that this feature makes them easily inhaled by humans, penetrating into the nasal mucosa.

However, there is still a dearth of information about the atmospheric distribution of pollen types and high-low risk periods for pollen allergy sufferers in Nigeria. Also, most aeropalynological studies have been conducted in the southern region, with little contributions from the Northcentral parts of the country but no record yet in other northern areas. This present study was carried out at selected study sites of North Western Nigeria, in order to examine the monthly depositional rate of airborne pollen and fern spores; investigate the effect of meteorological parameters on monthly airborne pollen load for a period of one year; observe the relationship between the recovered airborne pollen types and plants in the surrounding vegetation of the study sites. 


\section{Materials and Methods}

\section{Description of the study areas}

The study sites are located in Kaduna and Sokoto States, geographically positioned in the semi-arid zone of Northwestern Nigeria. Kaduna State is located on the southern end of the high plains of northern Nigeria, bounded by parallels of latitude $9^{\circ} 02^{\prime} \mathrm{N}$ and $11^{\circ} 32^{\prime} \mathrm{N}$, extending from the upper River Mariga on longitude $6^{\circ} 15^{\prime} \mathrm{E}$ of the Greenwich meridian on the foot slopes of the scarp of Jos Plateau. The rainy season has a short duration and occurs during the high sun period, followed by 5-7 months of dry season. The dry season is practically rainless and spans between October and April. The daily maximum temperatures show a major peak in April and a minor one in October. The mean minimum temperature rises from its lowest value of about $11{ }^{\circ} \mathrm{C}$ in December and January during harmattan, up to a high temperature of about $40{ }^{\circ} \mathrm{C}$ in July/August (Afolalu, 2009).

Sokoto State is located in the extreme part of this zone and geographically lies along latitude $13^{\circ} 05^{\prime} \mathrm{N}$ and longitude $05^{\circ} 15^{\prime} \mathrm{E}$. They are characterized by strong seasonality in rainfall and temperature distribution (Fadele et al., 2012; Yakubu et al., 2013). The wet season starts from late May and ends in early September but could extend to October with a mean annual rainfall of between $500 \mathrm{~mm}$ and 1300 $\mathrm{mm}$ with a peak rainfall in August. Dry season starts from October with the cold, dry dust-laden harmattan wind lasting up till February. Between the months of March to May, the daily temperatures are hot and dry, reaching $38^{\circ} \mathrm{C}$ $-42^{\circ} \mathrm{C}$ with relative humidity of less than $20 \%$ (Ahmed and Egwu, 2014).

In the two study areas, the vegetation type consists of Northern Guinea savannah and Sudano-Sahelian savannah (Afolalu, 2009; Ahmed and Egwu, 2014). The prolonged dry season (6-9 months) sustain fewer trees and grasses than the Southern Guinea savannah. The vegetation 183 undergone a severe destruction in the process of land clearing for the cultivation of important economic crops such as cotton, millet, maize and wheat. This is in addition to the devastation due to animal husbandry, especially cattle rearing which is remarkably favoured in this belt because the area is relatively free from tse-tse fly. The trees of the Sudan savannah include Acacia spp., Vitellaria paradoxa, Adansonia digitata and Ceiba pentandra, as cited by Yakubu et al. (2013). It is characterized by abundant grasses of less than $3 \mathrm{~m}$ height (Fadele et al., 2012) and few stunted trees often not taller than $15 \mathrm{~m}$. The main soil type is the ferruginous tropical soil mostly formed from the crystalline metamorphic rocks of granite gneiss (Afolalu, 2009).

\section{Study sites}

Two study sites were selected for aerosampling in the North-western Nigeria (Fig. 1) and a global positioning system was used to determine the position of the sampling points; Ahmadu Bello University, Zaria, Kaduna State (Latitude $11.0667^{\circ} \mathrm{N}$, Longitude $7.7000^{\circ} \mathrm{E}$ ) and Usman Danfodiyo University, Sokoto State (Latitude $9.945954^{\circ} \mathrm{N}$, Longitude $\left.8.893007^{\circ} \mathrm{E}\right)$.

\section{Plant enumeration}

This involved a qualitative enumeration of local plants in the surrounding vegetation using a quadrat of $25 \times 25 \mathrm{~m}$ plot for tree species and a quadrat of $1.0 \times 1.0 \mathrm{~m}$ plot for both herbs and grasses. Samples were collected randomly within each plot and plants were identified with the aid of keys, following the works of Dalziel (1937), Hutchinson and Dalziel (1954, 1963), Keay (1959), Keay et al. (1964) and Keay (1989). Voucher specimens of identified plants were collected and deposited at the University of Lagos Herbarium.

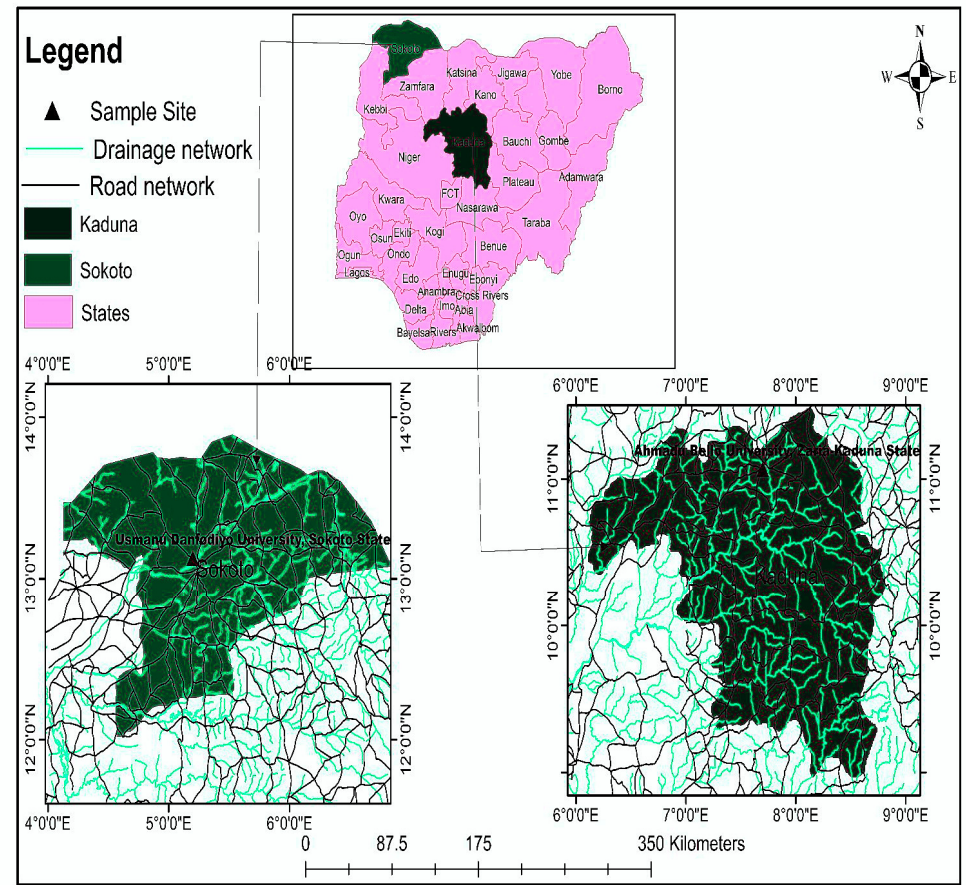

Fig. 1. Map of Nigeria showing the study sites in Kaduna and Sokoto States respectively 
184

\section{Setting up of pollen samplers}

A modified Tauber pollen sampler containing a solution of $50 \mathrm{ml}$ glycerol (to prevent drying up of organic particles), $10 \mathrm{ml}$ formaldehyde (to prevent the decay of dead organisms) and $5 \mathrm{ml}$ phenol (to kill insects) was constructed for the trapping of airborne palynomorphs at each of the study locations (Fig. 2), following the procedures of Adekanmbi and Ogundipe, 2010, Ezike et al., 2016 and Ajikah $e t$ al., 2017. This sampler was placed at the height of $5 \mathrm{ft}$. above the ground level (ca. average human height) with a $10 \mathrm{~cm}$ ring fixed at the top of it. In order to prevent it from being blown away by the wind, it was well cemented with one foot buried underground to provide support.

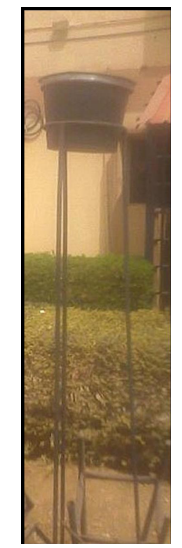

Fig. 2. A modified Tauber sampler mounted at one of the two study sites

\section{Sample collection}

The collection of residual solution was done monthly and spanned from July 2015 to June 2016, covering rainy and dry seasons. The sampler was washed at the end of each monthly collection.

\section{Treatment and microscopy}

In order to separate the residues containing palynomorphs from supernatant fluids, the solutions were centrifuged at 2,500 rpm for $5 \mathrm{~min}$. The residues were then subjected to acetolysis method of treatment following Erdtman (1969); preparation of acetolysis mixture involves an addition of concentrated sulphuric acid and acetic anhydride in the volume ratio of 1:9 respectively, $5 \mathrm{ml}$ of the prepared mixture was added to each sample residue and heated in a water bath at $100{ }^{\circ} \mathrm{C}$ for $10 \mathrm{~min}$. The residues were centrifuged, decanted, rinsed with distilled water and this process was carried out twice. The residues were stored in vials with an addition of two drops of glycerine for their preservation. Slide preparation and microscopy of prepared residues were also done. Identification of palynomorphs was achieved with the aid of photomicrographs in reference journals including Sowunmi (1973; 1995), Adekanmbi (2009), Adekanmbi and Ogundipe (2009), Gosling et al. (2013) and unpublished albums. Photomicrographs of some pollen and fern spore types (Fig. 3) were taken using a Euromex digital camera attached to an Olympus light microscope.

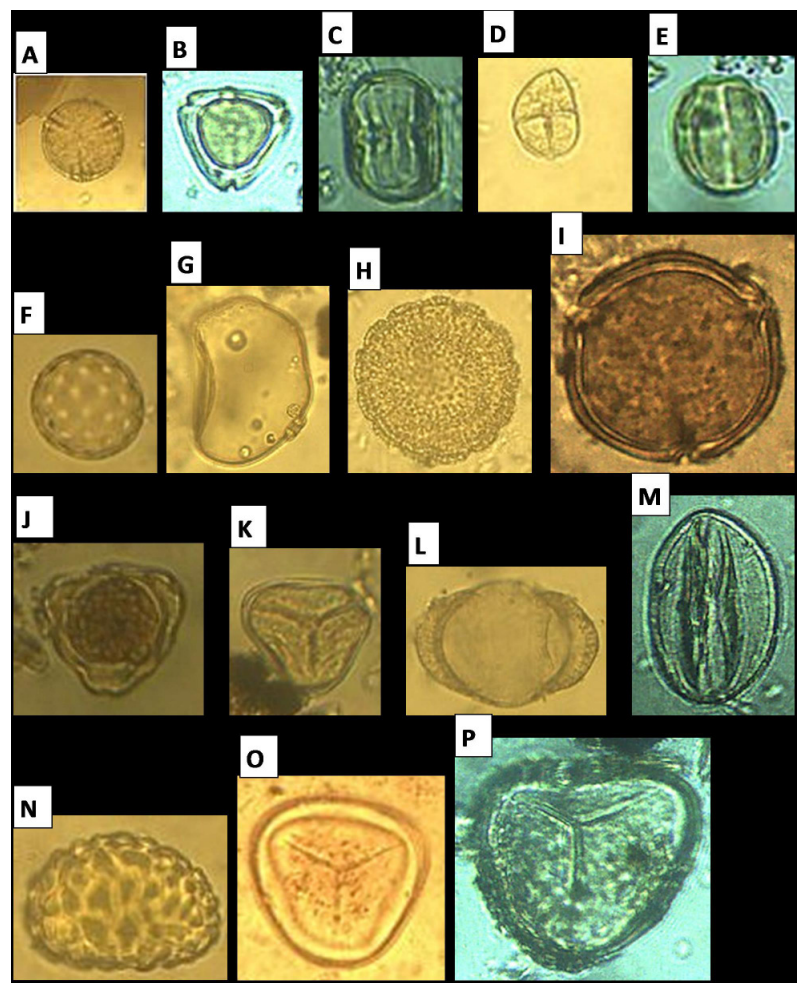

Fig. 3. Photomicrographs of some recovered pollen grains and fern spores in this study (A-M: Pollen grains; N-P: Fern spores); A. Alchornea laxiflora. B. Syzygium guineense C. Terminalia sp. D. Mimosa sp. E. Lannea/Sclerocarya sp. F. Amaranthus sp. G. Poaceae. H. Borreria sp. I. Senna sp. J. Casuarina equisetifolia. K. Elaeis guineensis. L. Pinus caribaea. M. Vitex sp. N. Nephrolepis sp. O. Pteris sp. P. Trilete fern. (All magnification: $\times 400$ objective) 


\section{Meteorological data collection}

Mean monthly data on air temperature, wind speed, relative humidity and rainfall for the sampling period in the study locations were sourced from the Nigerian Meteorological Agency, Oshodi, Lagos, Nigeria.

Correlation between monthly pollen concentrations and meteorological parameters

The relationship between monthly pollen concentrations and mean monthly meteorological data of the study locations was examined. Correlation coefficients were obtained by employing a Pearson correlation test at 95\% confidence interval, using SPSS statistic version 23 package, since these statistical data are parametric.

\section{Results}

\section{Palynological study}

All 29 atmospheric pollen types belonging to 17 different plant families were recorded in Ahmadu Bello University, Zaria, Kaduna State, Nigeria. Among these, 11, 7 and 11 were identified to familial, generic and species levels respectively. The unidentified pollen types were designated as pollen indeterminate. An annual pollen concentration of 2,723 was observed in this location. The highest total pollen count was recorded in the month of October $(25.30 \%)$ and a minimum in June $(0.96 \%)$. In Usmanu Danfodiyo University, Sokoto State Nigeria, 30 pollen types belonging to 18 different plant families were recorded. Among these, 11, 13 and 6 were identified to familial, generic and species levels respectively. An annual pollen concentration of 3,839 was observed in this location. The highest total pollen count was observed in March (47.85\%) and the lowest record in June (1.51\%).

The most abundant pollen types in Kaduna State were produced by Alchornea laxiflora, Pinus caribaea, Terminalia sp., Sapotaceae and Poaceae. The most abundant pollen types in Sokoto State were produced by Syzygium guineense, Alchornea sp., Sapotaceae and Poaceae. 26 airborne pollen types were common to both locations; $89.66 \%$ of pollen types identified in Kaduna state were also recovered in Sokoto state; $86.67 \%$ of pollen types identified in Sokoto state were also recovered in Kaduna state. In Kaduna State, highest monthly fern spore concentration of $6(28.57 \%)$ was observed in October and May respectively with no record in the months of July, August, December, January, February and March respectively. Of the three fern spore types recovered in this location, Nephrolepis sp. was the most abundantly represented, followed by Pteris sp. and a trilete fern spore respectively (Table 1). In Sokoto State, highest fern spore concentration of $35(60.35 \%)$ was observed in the month of November, followed by $19(32.76 \%)$ in the month of June. There was no record of fern spore count in the months of July, August, September, December, February and March respectively. A trilete fern spore was the most abundant, followed by Pteris sp. and Nephrolepis sp. respectively (Table 2). Monthly atmospheric pollen and fern spore concentrations of the study locations during the sampling period are presented in Tables 1 and 2.

Table 1. Frequency of atmospheric pollen and fern spores recovered from the study location in Kaduna State, Nigeria (July 2015 to June 2016)

\begin{tabular}{|c|c|c|c|c|c|c|c|c|c|c|c|c|c|c|}
\hline S. no & Pollen types & July & Aug. & Sep. & Oct. & Nov. & Dec. & Jan. & Feb. & Mar. & Apr. & May & June & Total \\
\hline 1. & Alchornea laxiflora & 88 & 2 & 7 & 18 & 0 & 26 & 3 & 0 & 0 & 0 & 0 & 0 & 144 \\
\hline 2. & Poaceae & 1 & 65 & 271 & 494 & 32 & 0 & 92 & 82 & 6 & 4 & 5 & 11 & 1,063 \\
\hline 3. & Amaranthus sp. & 0 & 5 & 43 & 33 & 3 & 4 & 8 & 6 & 1 & 2 & 1 & 0 & 106 \\
\hline 4. & Arecaceae & 0 & 0 & 3 & 7 & 0 & 4 & 5 & 0 & 0 & 0 & 0 & 0 & 19 \\
\hline 5. & Vitex sp. type & 0 & 11 & 23 & 50 & 0 & 0 & 15 & 0 & 0 & 0 & 0 & 0 & 99 \\
\hline 6. & Syzygium guineense & 0 & 42 & 12 & 19 & 6 & 10 & 9 & 9 & 12 & 2 & 1 & 3 & 125 \\
\hline 7. & Terminalia sp. & 0 & 4 & 12 & 1 & 0 & 143 & 2 & 235 & 4 & 18 & 0 & 0 & 419 \\
\hline 8. & $\begin{array}{l}\text { Casuarina } \\
\text { equisetifolia }\end{array}$ & 0 & 5 & 24 & 29 & 0 & 1 & 0 & 1 & 0 & 0 & 0 & 0 & 60 \\
\hline 9. & $\begin{array}{c}\text { Gomphrena } \\
\text { celosoides }\end{array}$ & 0 & 0 & 1 & 0 & 0 & 0 & 0 & 1 & 0 & 10 & 0 & 0 & 12 \\
\hline 10. & Euphorbiaceae & 0 & 3 & 1 & 2 & 1 & 0 & 0 & 0 & 0 & 0 & 0 & 0 & 7 \\
\hline 11. & Cyperaceae & 0 & 6 & 10 & 12 & 0 & 0 & 1 & 0 & 0 & 0 & 0 & 0 & 29 \\
\hline 12. & Elaeis guineensis & 0 & 22 & 4 & 0 & 7 & 0 & 3 & 2 & 1 & 0 & 0 & 0 & 39 \\
\hline 13. & Tridax procumbens & 0 & 0 & 0 & 6 & 5 & 4 & 1 & 16 & 0 & 0 & 0 & 3 & 35 \\
\hline 14. & Fabaceae & 0 & 1 & 7 & 0 & 4 & 8 & 28 & 36 & 2 & 0 & 3 & 4 & 93 \\
\hline
\end{tabular}




\begin{tabular}{|c|c|c|c|c|c|c|c|c|c|c|c|c|c|c|}
\hline 15. & Sapotaceae & 0 & 0 & 2 & 0 & 0 & 0 & 3 & 8 & 7 & 12 & 8 & 0 & 40 \\
\hline 16. & Acacia sp. & 0 & 1 & 2 & 2 & 1 & 0 & 1 & 1 & 0 & 0 & 0 & 0 & 8 \\
\hline 17. & $\begin{array}{c}\text { Lanneal } \\
\text { Sclerocaryasp. }\end{array}$ & 0 & 0 & 0 & 1 & 0 & 0 & 1 & 0 & 2 & 1 & 0 & 0 & 5 \\
\hline 18. & Pinus caribbea & 0 & 0 & 1 & 0 & 21 & 1 & 145 & 104 & 15 & 4 & 3 & 1 & 295 \\
\hline 19. & Borreria sp. & 0 & 0 & 1 & 15 & 5 & 0 & 2 & 3 & 1 & 1 & 0 & 0 & 28 \\
\hline 20. & Mimosaceae. & 0 & 0 & 0 & 0 & 0 & 0 & 0 & 1 & 1 & 1 & 0 & 1 & 4 \\
\hline 21. & Asystasia gagentica & 0 & 0 & 0 & 0 & 0 & 0 & 0 & 0 & 0 & 0 & 0 & 2 & 2 \\
\hline 22. & $\begin{array}{l}\text { Euphorbia } \\
\text { heterophylla }\end{array}$ & 0 & 0 & 0 & 0 & 0 & 0 & 11 & 0 & 0 & 0 & 2 & 0 & 13 \\
\hline 23. & $\begin{array}{c}\text { Nauclea latifolia } \\
\text { type }\end{array}$ & 0 & 0 & 0 & 0 & 0 & 0 & 2 & 0 & 0 & 0 & 0 & 0 & 2 \\
\hline 24. & Hyphaene sp. & 0 & 0 & 0 & 0 & 0 & 0 & 15 & 0 & 2 & 2 & 0 & 0 & 19 \\
\hline 25. & Senna sp. & 0 & 0 & 0 & 0 & 0 & 0 & 10 & 0 & 4 & 15 & 4 & 1 & 34 \\
\hline 26. & Convolvulaceae & 0 & 0 & 0 & 0 & 0 & 0 & 1 & 0 & 0 & 0 & 0 & 0 & 1 \\
\hline 27. & Asteraceae & 0 & 0 & 0 & 0 & 0 & 0 & 11 & 0 & 1 & 3 & 4 & 0 & 19 \\
\hline 28. & $\begin{array}{c}\text { Pollen } \\
\text { Indeterminate }\end{array}$ & 0 & 0 & 0 & 0 & 0 & 0 & 2 & 0 & 0 & 0 & 1 & 0 & 3 \\
\hline \multicolumn{2}{|c|}{$\begin{array}{l}\text { Total pollen count in each } \\
\text { month }\end{array}$} & 89 & 167 & 424 & 689 & 85 & 201 & 371 & 505 & 59 & 75 & 32 & 26 & 2,723 \\
\hline & $\%$ & 3.27 & 6.13 & 15.57 & 25.30 & 3.12 & 7.38 & 13.63 & 18.55 & 2.17 & 2.75 & 1.18 & 0.96 & \\
\hline \multicolumn{2}{|c|}{ Fern spore types } & & & & & & & & & & & & & Total \\
\hline 30. & Nephrolepis sp. & 0 & 0 & 1 & 4 & 0 & 0 & 0 & 0 & 0 & 2 & 1 & 1 & 9 \\
\hline 31. & Pteris sp. & 0 & 0 & 0 & 1 & 2 & 0 & 0 & 0 & 0 & 0 & 2 & 1 & 6 \\
\hline 32. & Trilete fern spore & 0 & 0 & 0 & 1 & 0 & 0 & 0 & 0 & 0 & 0 & 3 & 2 & 6 \\
\hline & fern spore count & 0 & 0 & 1 & 6 & 2 & 0 & 0 & 0 & 0 & 2 & 6 & 4 & 21 \\
\hline & $\%$ & 0.00 & 0.00 & 4.76 & 28.57 & 9.52 & 0.00 & 0.00 & 0.00 & 0.00 & 9.52 & 28.57 & 19.05 & \\
\hline
\end{tabular}

Table 2. Frequency of atmospheric pollen and fern spores recovered from the study location in Sokoto State, Nigeria (July 2015 to June 2016)

\begin{tabular}{|c|c|c|c|c|c|c|c|c|c|c|c|c|c|c|}
\hline S. no & Pollen types & July & Aug. & Sep. & Oct. & Nov. & Dec. & Jan. & Feb. & Mar. & Apr. & May & June & Total \\
\hline 1. & Poaceae & 47 & 37 & 195 & 320 & 182 & 68 & 92 & 27 & 183 & 11 & 26 & 9 & 1,197 \\
\hline 2. & Asteraceae & 7 & 0 & 0 & 0 & 0 & 0 & 0 & 0 & 1 & 0 & 0 & 0 & 8 \\
\hline 3. & Terminalia sp. & 1 & 1 & 2 & 1 & 5 & 4 & 2 & 2 & 4 & 0 & 5 & 10 & 37 \\
\hline 4. & Borreria sp. & 0 & 0 & 6 & 0 & 0 & 0 & 0 & 0 & 4 & 0 & 2 & 0 & 12 \\
\hline
\end{tabular}




\begin{tabular}{|c|c|c|c|c|c|c|c|c|c|c|c|c|c|c|}
\hline 5. & Vitex sp. type & 0 & 5 & 0 & 0 & 0 & 0 & 0 & 9 & 1 & 0 & 0 & 0 & 15 \\
\hline 6. & Sapotaceae & 0 & 1 & 0 & 1 & 2 & 2 & 7 & 3 & 149 & 125 & 20 & 0 & 310 \\
\hline 7 & Mimosa sp. & 1 & 0 & 2 & 0 & 1 & 0 & 0 & 0 & 9 & 0 & 2 & 6 & 21 \\
\hline 8. & Syzygium guineense & 0 & 1 & 6 & 5 & 15 & 0 & 5 & 184 & 1,433 & 123 & 2 & 1 & 1,775 \\
\hline 9. & Alchornea sp. & 4 & 136 & 7 & 5 & 118 & 3 & 7 & 4 & 0 & 0 & 2 & 0 & 286 \\
\hline 10. & Amaranthus sp. & 0 & 3 & 11 & 3 & 5 & 2 & 0 & 0 & 3 & 0 & 0 & 0 & 27 \\
\hline 11. & $\begin{array}{l}\text { Casuarina } \\
\text { equisetifolia }\end{array}$ & 0 & 1 & 1 & 0 & 0 & 0 & 0 & 0 & 0 & 0 & 0 & 0 & 2 \\
\hline 12. & Cyperaceae & 0 & 1 & 2 & 3 & 1 & 0 & 0 & 0 & 0 & 0 & 0 & 0 & 7 \\
\hline 13. & Acacia sp. & 0 & 2 & 1 & 2 & 0 & 0 & 0 & 0 & 3 & 0 & 0 & 0 & 8 \\
\hline 14. & Tridax procumbens & 0 & 0 & 0 & 1 & 2 & 1 & 1 & 0 & 5 & 0 & 3 & 18 & 31 \\
\hline 15. & Elaeis guineensis & 0 & 0 & 0 & 0 & 27 & 0 & 3 & 0 & 0 & 0 & 1 & 0 & 31 \\
\hline 16. & Gomphrena celosoides & 0 & 0 & 0 & 0 & 0 & 0 & 0 & 0 & 3 & 1 & 0 & 0 & 4 \\
\hline 17. & Hyphaene sp. & 0 & 0 & 0 & 0 & 0 & 0 & 0 & 0 & 1 & 0 & 1 & 0 & 2 \\
\hline 18. & Senna sp. & 0 & 0 & 0 & 0 & 0 & 0 & 0 & 0 & 16 & 0 & 0 & 12 & 28 \\
\hline 19. & Calystegia sp. type & 0 & 0 & 0 & 0 & 0 & 0 & 0 & 0 & 2 & 0 & 0 & 0 & 2 \\
\hline 20. & Citrus sp. & 0 & 0 & 0 & 0 & 0 & 0 & 0 & 0 & 1 & 0 & 0 & 0 & 1 \\
\hline 21. & Uapaca sp. & 0 & 0 & 0 & 0 & 0 & 0 & 0 & 0 & 1 & 0 & 1 & 0 & 2 \\
\hline 22. & Nauclea sp. type & 0 & 0 & 0 & 0 & 0 & 0 & 0 & 0 & 3 & 1 & 0 & 0 & 4 \\
\hline 23. & $\begin{array}{c}\text { Lannea / Sclerocarya } \\
\text { sp. }\end{array}$ & 0 & 0 & 0 & 0 & 0 & 0 & 0 & 0 & 0 & 1 & 2 & 0 & 3 \\
\hline 24. & Convolvulaceae & 0 & 0 & 0 & 0 & 0 & 0 & 0 & 0 & 2 & 0 & 0 & 0 & 2 \\
\hline 25. & Tiliaceae & 0 & 0 & 0 & 0 & 0 & 0 & 0 & 0 & 1 & 0 & 0 & 0 & 1 \\
\hline 26. & Fabaceae & 2 & 0 & 0 & 0 & 1 & 0 & 0 & 0 & 0 & 2 & 0 & 0 & 5 \\
\hline 27. & Arecaceae & 0 & 0 & 0 & 0 & 1 & 0 & 0 & 0 & 5 & 0 & 1 & 0 & 7 \\
\hline 28. & Euphorbiaceae & 0 & 0 & 0 & 0 & 0 & 0 & 0 & 0 & 14 & 0 & 0 & 1 & 15 \\
\hline 29. & Mimosaceae & 0 & 0 & 0 & 0 & 0 & 0 & 0 & 0 & 0 & 2 & 0 & 1 & 3 \\
\hline 30. & Pollen Indeterminate & 0 & 0 & 0 & 0 & 0 & 0 & 0 & 0 & 2 & 0 & 0 & 0 & 2 \\
\hline \multicolumn{2}{|c|}{$\begin{array}{l}\text { Total pollen count in each } \\
\text { month }\end{array}$} & 62 & 188 & 233 & 341 & 360 & 80 & 117 & 229 & 1837 & 266 & 68 & 58 & 3,839 \\
\hline \multicolumn{2}{|r|}{$\%$} & 1.62 & 4.90 & 6.07 & 8.88 & 9.38 & 2.08 & 3.05 & 5.97 & 47.85 & 6.93 & 1.77 & 1.51 & \\
\hline \multicolumn{2}{|r|}{ Fern spore types } & & & & & & & & & & & & & Total \\
\hline 32. & Nephrolepis sp. & 0 & 0 & 0 & 1 & 6 & 0 & 1 & 0 & 0 & 0 & 0 & 3 & 11 \\
\hline 33. & Pteris sp. & 0 & 0 & 0 & 0 & 13 & 0 & 0 & 0 & 0 & 1 & 0 & 8 & 22 \\
\hline 34. & Trilete fern spore & 0 & 0 & 0 & 0 & 16 & 0 & 0 & 0 & 0 & 0 & 1 & 8 & 25 \\
\hline & al fern spore count & 0 & 0 & 0 & 1 & 35 & 0 & 1 & 0 & 0 & 1 & 1 & 19 & 58 \\
\hline & $\%$ & 0.00 & 0.00 & 0.00 & 1.72 & 60.35 & 0.00 & 1.72 & 0.00 & 0.00 & 1.72 & 1.72 & 32.76 & \\
\hline
\end{tabular}


188

Vegetation description and meteorological data of the study locations

The vegetation in Ahmadu Bello University, Zaria, Kaduna State is a northern guinea savannah type with sparsely distributed trees and also dominated by shrubs. The vegetation in Usmanu Danfodiyo University, Sokoto State is a typical Sahel savannah type with sparsely dispersed trees, characterized by a fissured thick bark layer. The entire vegetation predominantly consists of unidentified grasses which do not exceed a height of $1-2 \mathrm{~cm}$. Identified plants in the study locations are listed in Tables 3 and 4 . Variations were observed in the monthly mean values of meteorological parameters including air temperature, rainfall, relative humidity and wind speed at the study sites during the sampling period (Figs. 4-7).

Table 3. Enumerated plants around the study site in Ahmadu Bello University, Kaduna State

\begin{tabular}{|c|c|c|c|}
\hline S. no & Scientific name & Family name & Habit \\
\hline 1. & Pennisetum sp. & Poaceae & Grass \\
\hline 2. & Tridax procumbens $\mathrm{L}$. & Asteraceae & Herb \\
\hline 3. & Ageratum conyzoides $\mathrm{L}$. & Asteraceae & Herb \\
\hline 4. & Senna occidentalis $\mathrm{L}$. & Fabaceae & Shrub \\
\hline 5. & Alchornea laxiflora (Benth.) Pax \& K. Hoffm. & Euphorbiaceae & Shrub \\
\hline 6. & Albizia lebbeck (L.) Benth. & Mimosaceae & Tree \\
\hline 7. & Acacia sieberiana DC. & Fabaceae & Tree \\
\hline 8. & Azadirachta indica A. Juss. & Meliaceae & Tree \\
\hline 9. & Cassia sieberiana $\mathrm{DC}$. & Fabaceae & Tree \\
\hline 10. & Cassia sp. & Fabaceae & Tree \\
\hline 11. & Acacia seyal Del. & Fabaceae & Tree \\
\hline 12. & Pinus caribaea Morlet. & Pinaceae & Tree \\
\hline 13. & Khaya grandifoliola (Welw.) C.DC. & Meliaceae & Tree \\
\hline 14. & Cyperus sp. & Cyperaceae & Sedge \\
\hline
\end{tabular}

Table 4. Enumerated plants around the study site in Usmanu Danfodiyo University, Sokoto State

\begin{tabular}{cccc}
\hline S. no & Scientific name & Family names & Habit \\
\hline 1. & Tridaxprocumbens L. & Asteraceae & Herb \\
\hline 2. & Euphorbia hirta L. & Euphorbiaceae & Shrub \\
\hline 3. & Sclerocarya birrea (A Rich.) Hochst. & Anacardiaceae & Tree \\
4. & Diospyros mespiliformis (Hochst. ex A.DC.) & Ebenaceae & Tree \\
5. & Azadirachta indica A. Juss. & Meliaceae & Tree \\
\hline 6. & Borassus aethiopium Mart. & Arecaceae & Tree \\
\hline
\end{tabular}

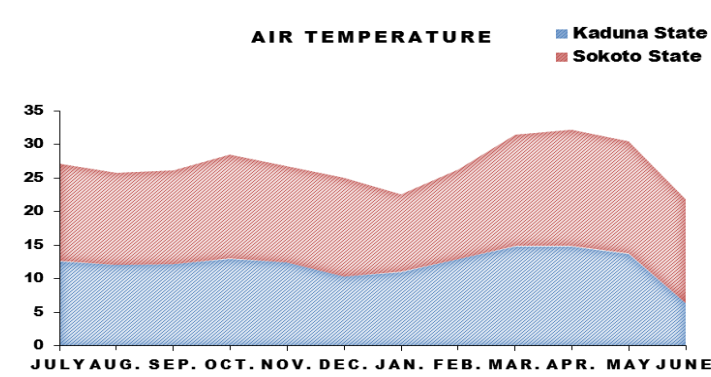

Fig. 4. Mean monthly air temperature values $\left({ }^{\circ} \mathrm{C}\right)$ in Kaduna and Sokoto States

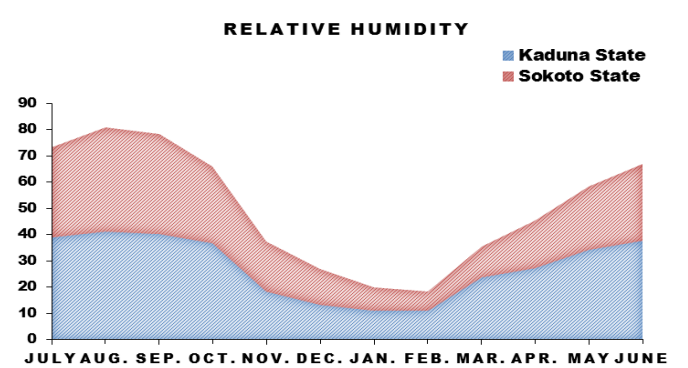

Fig. 5. Mean monthly relative humidity values (\%) in Kaduna and Sokoto States 


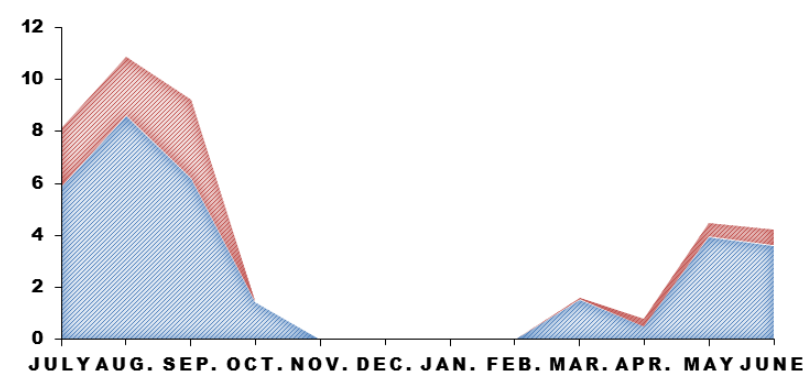

Fig. 6. Mean monthly rainfall values $(\mathrm{mm})$ in Kaduna and Sokoto States

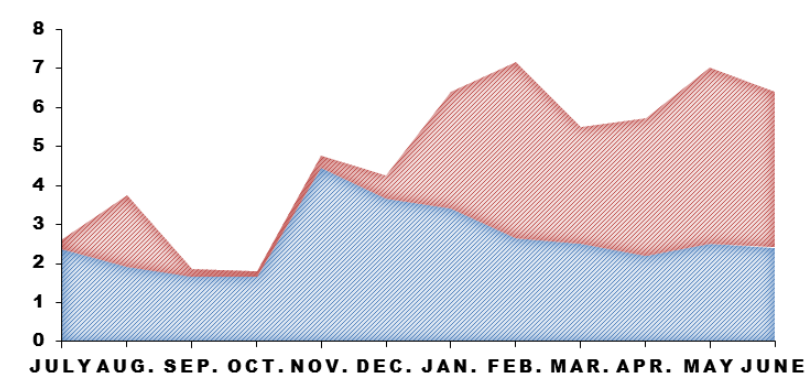

Fig. 7. Mean monthly wind speed values (knot) in Kaduna and Sokoto States

Table 5. Relationship between monthly pollen concentrations and values of mean meteorological parameters at the study site in Kaduna state at $95 \%$ confidence interval $(\mathrm{P}<0.05)$

\begin{tabular}{|c|c|c|c|c|c|c|}
\hline & & Pollen concentration & Temperature & Wind speed & Relative humidity & Rainfall \\
\hline \multirow{5}{*}{ Pearson correlation } & Pollen concentration & 1.000 & -.240 & -.374 & .266 & .009 \\
\hline & Temperature & -.240 & 1.000 & -.421 & .117 & -.160 \\
\hline & Wind speed & -.374 & -.421 & 1.000 & -.741 & -.523 \\
\hline & Relative humidity & .266 & .117 & -.741 & 1.000 & .820 \\
\hline & Rainfall & .009 & -.160 & -.523 & .820 & 1.000 \\
\hline \multirow{5}{*}{ Sig. (1-tailed) } & Pollen concentration & . & .252 & .143 & .229 & .490 \\
\hline & Temperature & .252 & . & .113 & .374 & .330 \\
\hline & Wind speed & .143 & .113 & . & .007 & .060 \\
\hline & Relative humidity & .229 & .374 & .007 & . & .002 \\
\hline & Rainfall & .490 & .330 & .060 & .002 & . \\
\hline \multirow{5}{*}{$\mathbf{N}$} & Pollen concentration & 10 & 10 & 10 & 10 & 10 \\
\hline & Temperature & 10 & 10 & 10 & 10 & 10 \\
\hline & Wind speed & 10 & 10 & 10 & 10 & 10 \\
\hline & Relative humidity & 10 & 10 & 10 & 10 & 10 \\
\hline & Rainfall & 10 & 10 & 10 & 10 & 10 \\
\hline
\end{tabular}

Table 6. Correlation coefficients showing the significance levels in the correlation between monthly pollen concentrations and values of mean monthly meteorological parameters at the study site in Kaduna State at 95\% confidence interval $(\mathrm{P}<0.05)$

\begin{tabular}{|c|c|c|c|c|c|c|c|c|}
\hline \multirow{2}{*}{ Model } & \multicolumn{2}{|c|}{ Unstandardized coefficients } & \multirow{2}{*}{$\begin{array}{c}\text { Standardized } \\
\text { coefficients } \\
\text { Beta }\end{array}$} & \multirow[b]{2}{*}{$\mathrm{T}$} & \multirow[b]{2}{*}{ Sig. } & \multicolumn{3}{|c|}{ Correlations } \\
\hline & B & Std. Error & & & & Zero-order & Partial & Part \\
\hline (Constant) & 2146.784 & 956.253 & & 2.245 & .075 & & & \\
\hline Temperature & -65.376 & 26.118 & -.826 & -2.503 & .054 & -.240 & -.746 & -.650 \\
\hline Wind speed & -94.789 & 52.733 & -.803 & -1.798 & .132 & -.374 & -.627 & -.467 \\
\hline Relative humidity & 6.965 & 6.347 & .650 & 1.097 & .322 & .266 & .441 & .285 \\
\hline Rainfall & -1.254 & .592 & -1.076 & -2.119 & .088 & .009 & -.688 & -.550 \\
\hline
\end{tabular}


Table 7. Relationship between monthly pollen concentrations and values of mean meteorological parameters at the study site in Sokoto state at $95 \%$ confidence interval $(\mathrm{P}<0.05)$

\begin{tabular}{|c|c|c|c|c|c|c|}
\hline & & Pollen concentration & Temperature & Wind speed & Relative humidity & Rainfall \\
\hline \multirow{5}{*}{ Pearson correlation } & Pollen concentration & 1.000 & .088 & .005 & -.011 & -.123 \\
\hline & Temperature & .088 & 1.000 & -.694 & .419 & .286 \\
\hline & Wind speed & .005 & -.694 & 1.000 & -.595 & -.452 \\
\hline & Relative humidity & -.011 & .419 & -.595 & 1.000 & .949 \\
\hline & Rainfall & -.123 & .286 & -.452 & .949 & 1.000 \\
\hline \multirow{5}{*}{ Sig. $(1$-tailed $)$} & Pollen concentration & . & .426 & .496 & .490 & .396 \\
\hline & Temperature & .426 & . & .042 & .175 & .267 \\
\hline & Wind speed & .496 & .042 & . & .080 & .155 \\
\hline & Relative humidity & .490 & .175 & .080 & . & .001 \\
\hline & Rainfall & .396 & .267 & .155 & .001 & . \\
\hline \multirow{5}{*}{$\mathbf{N}$} & Pollen concentration & 7 & 7 & 7 & 7 & 7 \\
\hline & Temperature & 7 & 7 & 7 & 7 & 7 \\
\hline & Wind speed & 7 & 7 & 7 & 7 & 7 \\
\hline & Relative humidity & 7 & 7 & 7 & 7 & 7 \\
\hline & Rainfall & 7 & 7 & 7 & 7 & 7 \\
\hline
\end{tabular}

Table 8. Correlation coefficients showing the significance levels in the correlation between monthly pollen concentrations and values of mean monthly meteorological parameters at the study site in Sokoto state at $95 \%$ confidence interval $(\mathrm{P}<0.05)$

\begin{tabular}{|c|c|c|c|c|c|c|c|c|}
\hline \multirow{2}{*}{ Model } & \multicolumn{2}{|c|}{ Unstandardized coefficients } & \multirow{2}{*}{$\begin{array}{c}\begin{array}{c}\text { Standardized } \\
\text { coefficients }\end{array} \\
\text { Beta }\end{array}$} & \multirow[b]{2}{*}{$\mathrm{T}$} & \multirow[b]{2}{*}{ Sig. } & \multicolumn{3}{|c|}{ Correlations } \\
\hline & B & Std. Error & & & & Zero-order & Partial & Part \\
\hline (Constant) & -92.192 & 1278.517 & & -.072 & .949 & & & \\
\hline Temperature & 4.267 & 44.693 & .087 & .095 & .933 & .088 & .067 & .062 \\
\hline Wind speed & 9.126 & 32.909 & .294 & .277 & .808 & .005 & .192 & .180 \\
\hline Relative humidity & 5.271 & 9.579 & 1.419 & .550 & .637 & -.011 & .363 & .357 \\
\hline Rainfall & -1.708 & 2.917 & -1.362 & -.585 & .618 & -.123 & -.382 & -.379 \\
\hline
\end{tabular}

\section{Discussion}

The results of the present study reveal the atmospheric distribution of different pollen types recovered from the two sampling locations (Tables 1 and 2). Previous studies conducted in Nigeria have similarly employed the use of gravitational and non-volumetric samplers for the trapping of airborne pollen and spores (Njokuocha, 2006; Adekanmbi and Ogundipe, 2010; Essien and Aina, 2014; Adeniyi et al., 2014; Ezike et al., 2016; Ajikah et al., 2017; Adekanmbi et al., 2017; Alebiosu et al., 2017). Higher monthly pollen concentrations were observed from October to March and this is similar to Njokuocha (2006) who reported more pollen load from September to January in Nsukka, South-Eastern, Nigeria. These findings are also supported by Ezike et al. (2016) who observed highest pollen abundance from October to January in Garki, Abuja, North-Central Nigeria, during which they designated these harmattan months as a period of higher risk for individuals that are hypersensitive to pollen grains. In contrast to these findings, Essien and Aina (2014) reported monthly pollen concentration to be more abundant in the month of May, followed by June.

It is noteworthy that a considerable similarity exists in the array of pollen types recovered from the two locations (Tables 1 and 2). This remarkable similarity may be attributed to a wide geographical diversity of parent plants producing these pollen types in vegetation both proximal and distal to the two study sites. Preponderant pollen types identified in this study are similar to Ajikah et al. (2015) who reported Combretaceae, Sapotaceae and Poaceae as major airborne pollen producers in their study. The dominant pollen recovery of Pinus caribaea in Kaduna State corresponds with Singh and Dahiya (2008), Scevkova et al. (2015) who identified a species of Pinus as one of the most abundant atmospheric pollen types in their studies. Findings from this study agree well with Essien and Agwu (2013) who reported that pollen of Syzygium guineense and Combretaceae were abundant in the atmosphere of their study location. The atmospheric dominance of Poaceae pollen at the study sites is supported by Njokuocha (2006), Essien and Agwu (2013), Adeniyi et al. (2014) and Ezike et al. (2016) who previously observed the predominance of Poaceae pollen in their respective studies.

However, there were variations in the abundance of airborne Poaceae pollen in the locations over the study period (Tables 1 and 2). Essien and Aina (2014) cited Suphioglu et al. (1992), stating that airborne Poaceae pollen 
consists of allergens capable of releasing microscopic particles into the atmosphere after dehiscence. The incidence of atmospheric Poaceae pollen is globally most important as a trigger of allergic reactions and has been implicated in pollinosis, as remarked by Taketomi et al. (2008). Scevkova et al. (2015) detected sensitization to Poaceae pollen as most frequent among pollen allergens identified in the atmosphere of Bratislavia, Slovakia. Galant et al. (2010) reported higher prevalence of allergic subjects with positive responses to grasses (Poaceae) compared with weeds, trees, molds and other sources of aeroallergens in California, U.S.A. Singh and Dahiya (2008) carried out a clinico-immunological survey in different parts of India. In their survey, Poaceae was identified as one of the most important allergens in Kanpur and Central India.

The greater recovery of Nephrolepis sp. in Kaduna State (Table 1), is similar to earlier findings of Njokuocha (2006) who identified the spore of Nephrolepis biserrata as one of the most abundantly recovered fern spore types in his study. The greater recovery of a trilete fern spore in Sokoto State (Table 2) corresponds with the findings of Essien et al. (2014) who reported highest abundance of a trilete fern spore, followed by Pteris dentata and Pteris sp. in their study. The highest recovery of fern spores in October and November at the respective study sites in Kaduna and Sokoto States, agrees with Njokuocha (2006) who recorded higher monthly fern spore concentrations in the respective months of September and November. The recovery of these fern spore types indicates that the study locations are bounded by an established fresh water swamp forest; a parent vegetation of the pteridophytes, inhabited by the represented fern plants in the surrounding environment of the two study sites.

No statistically significant correlation was observed between monthly pollen concentrations and mean values of meteorological parameters at the two study locations (Tables 6 and 8). A positive relationship was observed between monthly pollen concentrations and meteorological parameters including relative humidity and rainfall, but negatively with air temperature and wind speed in Kaduna State (Table 5). This is in agreement with Ezike et al. (2016) who reported that monthly pollen load was positively related with relative humidity but negatively with rainfall, wind speed and air temperature. On the other hand, a negative relationship was observed between monthly pollen concentrations and meteorological parameters including relative humidity and rainfall, but positively with air temperature in Sokoto State (Table 7). This is similar to the findings of Abdulrahaman et al. (2015) who earlier reported that monthly pollen concentrations were positively related with temperature. Ajikah et al. (2015) recorded a negative relationship between monthly pollen load and relative humidity. Njokuocha (2006) also found that monthly pollen load were positively related with temperature but negatively with rainfall. It is expedient to know that an inverse meteorological relationship existed between monthly pollen concentrations of the two locations during the study period (Tables 6 and 8).

Airborne pollen of some plants identified in the immediate vegetation of the two locations were recovered in this study. In Kaduna state, parent plants of the recovered pollen types of Alchornea laxiflora, Tridax procumbens,
Pinus caribaea, Albizia sp., Acacia sp., Poaceae, Fabaceae, Asteraceae and Cyperaceae, can be identified in the immediate vegetation of the study site. Other enumerated plants including Pennisetum sp. and some unidentified grass species belong to Poaceae; Cyperus spp. belong to Cyperaceae; Ageratum conyzoides belong to Asteraceae (Table 3). In Sokoto state, parent plants of the recovered pollen types of Tridax procumbens and Poaceae can be identified in the surrounding vegetation of the study site. (Table 4). These findings are supported by Njokuocha (2006) and Adeonipekun (2012) who observed that pollen from the local vegetation of their sampling areas were considerably recovered in their respective studies. This however confirms a remarkable relationship between the recovered airborne pollen spectra and plants identified in the surrounding vegetation of the study locations.

\section{Conclusions}

Pollen peak period from October to March is designated as high risk period for pollen hypersensitive individuals at the study sites in Kaduna and Sokoto states respectively. The results have shown that pollen allergy sufferers in these study locations are exposed to considerably similar types of atmospheric pollen grains, consequent of which they may experience same allergic conditions, including allergic rhinitis, atopic dermatitis among others in these locations. Further clinical studies on the allergenicity of preponderant airborne pollen grains would guide health care providers in making clinical diagnosis and aid in the treatment of patients reporting for pollen allergy in Nigeria. It will also help pollen allergy sufferers to identify plants producing allergenic pollen in their environment and adopt prophylactic measures against this menace.

\section{Acknowledgements}

The financial support received from TETfund, Nigeria for the successful completion of this research is greatly appreciated. The authors sincerely thank Mr. Nemadi (Ahmadu Bello University, Kaduna State) and Mr. Hamidu (Usman Danfodiyo University, Sokoto State) for their immense assistance during sample collection.

\section{References}

Abdulrahaman A, Aruofor OS, Garuba T, Kolawole OS, Olahan GS, Oladele FA (2015). Aeropalynological investigation of the University of Ilorin, Ilorin, Nigeria. Journal of Applied Science and Environmental Management 19(1):53-63.

Abdulrahaman A, Aruofor OS, Garuba T, Kolawole OS, Olahan GS, Oladele FA (2015). Aeropalynological investigation of the University of Ilorin, Ilorin, Nigeria. Journal of Applied Science and Environmental Management 19(1):53-63.

Adekanmbi OH (2009). Pollen grains of Asteraceae and analogous echinate grains. International Journal of Botany 5(4):295-300.

Adekanmbi OH, Ogundipe OT (2009). Pollen grains of Lagos lagoon swamp and hinter-land vegetation-1. International Journal of Botany 5:270-278.

Adekanmbi OH, Ogundipe OT (2010). Aeropalynological studies of the University of Lagos campus, Nigeria. Notulae Scientia Biologicae 2(4):3439. 
192

Adekanmbi OH, Alebiosu OS, Ajikah LB (2017). A survey of atmospheric palynomorphs in relation to weather and vegetation at selected study sites in South Southern Nigeria. Nigerian Journal of Botany 30(2):153169.

Adeniyi TA, Adeonipekun PA, Olowokudejo JD, Idowu SA (2014). Airborne pollen records of Shomolu local government area in Lagos State. Notulae Scientia Biologicae 6(4):428-432.

Adeonipekun AP, John M (2011). Palynological investigation of haze dust in Ayetoro-Itele Ota, South West Nigeria. Journal of Ecology and the Natural Environment 3(14):455-460.

Adeonipekun AP (2012). Comparative aeropalynology of Ota, Nigeria. Journal ofEcology and the Natural Environment 4(12):314320.

Afolalu FO (2009). Geo-environmental impact assessment of effluent water disposal on underground water in parts of Samaru, Zaria, Kaduna State, Nigeria. The Pacific Journal of Science and Technology 10(1):581-590.

Agwu COC, OsibeEE (1992). Airborne palynomorphs of Nsukka during the monthsofFebruary-April 1990. Nigerian Journal ofBotany 5:177-185.

Ahmed A, Egwu GO (2014). Management practices and constraints of sheep farmers in Sokoto State, North-western Nigeria. International Journal of Science, Environment and Technology 3(2):735-748.

Ajikah L, Ogundipe OT, Bamgboye O (2015). Palynological survey of airborne pollen and spores in the University of Lagos, Akoka campus, South-western Nigeria. Ife Journal of Science 17(3):643-655.

Ajikah LB, Alebiosu OS, Adekanmbi OH, Oshinlaja EO, Ogundipe OT (2017). Aeropalynological investigation of three local governments in Lagos, South West Nigeria. Nigerian Journal of Botany 30(7):107-118.

Alebiosu OS, Adekanmbi OH, Nodza GI, Ogundipe OT (2017). Aeropalynological study of two selected locations in North-Central Nigeria. Aerobiologia 34(2):187-202.

Dalziel JM (1937). The useful plants of West tropical Africa. Crown Overseas Agents for the Colonies, London.

D'Amato G, Liccard G, D'Amato M, Cazzola M (2002). Respiratory allergic diseases induced by outdoor air pollution in urban areas. Respiratory Medicine 57(3):161-163.

Essien BC, Agwu COC (2013). Aeropalynological study of Anyigba, Kogi State, Nigeria. Standard Scientific Research andEssays 1(13):347-351.

Essien BC, Aina DO (2014). The role of airborne pollen grains of some angiosperms and fungal spores in allergic and pathogenic infections in Anyigba, Kogi state, Nigeria. International Journal of Advances in Medical Sciences and Biotechnology 2(3):23-28.

Essien BC, Agwu COC, Taiga A (2014). A comparative analytical investigation of airborne palynomorphs within Kogi State University Teaching Hospital, Anyigba, Nigeria. Journal of Applied Sciences and Research 2(1):136-142.

Erdtman G(1969). Handbook of palynology. Munksgaard, Copenhagen.

Ezike DN, Nnamani CV, Ogundipe OT, Adekanmbi OH (2016). Airborne pollen and fungal spores in Garki, Abuja (North-Central Nigeria).Aerobiologia 32(4):697-707.

Fadele SI, Jatau BS, Patrick NO (2012). Subsurface stratigraphic mapping using the DC electrical resistivity around Shika, Kaduna State, Nigeria. International Journal of Engineering Research and Applications 2(5):11541161.

Galant S, Berger W, Gillman S, Goldsobel A (2010). Prevalence of sensitization to aeroallergens in California patients with respiratory allergy. Annals of Allergy, Asthma and Immunology 81:203-210.
Gosling WD, Miller CS, Livingstone DA (2013). Atlas of the tropical West Africa pollen flora. Review of Paleobotany and Palynology 199:1-135.

Hutchinson JB, Dalziel JM (1954). Flora of West Tropical Africa. In: Keay RWJ (Ed). Crown Agents for Oversea Governments and Administration, London.

Hutchinson JB, Dalziel JM (1963). Flora of West Tropical Africa. In: Hepper FN (Ed). Crown Agents for Oversea Governments and Administration, London.

Kamijo S, Takai T, Kuhara T, Kokura T, Ushio A, Ota M, et al., Okumura $\mathrm{K}$ (2013). Cupressaceae pollen grains modulate dendritic cell response and exhibit IgE inducing adjuvant activity in vivo. The Journal of Immunology 183:6087-6094.

Karalyan ZA, Ter-Pogossyan ZR, Abroyan LO, Hakobyan LH, Avetisyan AS, Karalyan NY,KaralovaEM(2016). Characterization of the atypical lymphocytes in African swine fever. Veterinary World 9(7):792-800.

Keay RWJ (1959). An Outline of Nigerian Vegetation. Government Printer $\left(2^{\text {nd }}\right.$ ed), Lagos, Nigeriapp 146.

Keay RWJ (1989). Trees of Nigeria Clarendon Press Oxford, London pp 19-30.

Keay RWJ, Onochie CFA, Standfield DP (1964). Nigerian trees. Department of Forest Research(Vol. 1), Ibadan, Nigeria.

Knapp WA (1971). A montane pollen species from the upper tertiary of the Niger Delta. Journal of Mineralogy and Geology 6:23-29.

Njokuocha RC (2006). Airborne pollen grains in Nsukka, Nigeria. Grana 45:73-80.

Ščevkova J, Dušička J, Hrubiško M, Mičieta K (2015). Influence of airborne pollen counts and length of pollen season length of selected allergenic plants on the concentration of sIgE antibodies on the population of Bratislava, Slovakia. Annals of Agricultural and Environmental Medicine 22(3):451-455.

Singh AB, Rawat A (2000). Aeroallergens in India. In: Prasad R (Ed). Advances in Allergy and Asthma. Shivam Publication, Lucknow, India.

Singh AB, Dahiya P (2008). Aerobiological researches on pollen and fungi in India during the last fifty years: An overview. Indian Journal of Allergy and Asthma Immunology 22(1):27-38.

Singh AB, Kumar P (2004). Aerial pollen diversity in India and their clinical significance in allergic diseases. Indian Journal of Clinical Biochemistry 19(2):190-201.

Singh $A B$, Mathur C (2012). An aerobiological perspective in allergy and asthma. Asia Pacific Allergy 2(3):210-222.

Sokoto MB, Tanko L, Abubakar L, Dikko AU, Abdullahi YM (2016). Effect of climate variables on major cereal crops production in Sokoto State, Nigeria. American Journal of Experimental Agriculture 10(3):1-7.

SowunmiMA(1973). Pollen grains of Nigerian plants. Grana 13(3):145-186.

Sowunmi MA (1995). Pollen of Nigerian plants. Grana 34(2):120-141.

Suphioglu C, Singh MB, Taylor P, Bellomo R, Holmes P, Puy R, Knox RB (1992). Mechanism of grass-pollen induced asthma. Lancet 339:569572.

Taketomi EA, Sopelete MC, Moreira PFDS, Vieira FDAM (2006). Pollen allergic disease: pollens and its major allergens. Revista Brasileira de Otorrinolaringologia 72(4):562-567.

Yakubu DH, Abubakar BZ, Atala TK, Muhammed A, Abdullahi MK (2013). Assessing the effects of socio-economic factors on ICTs adoption among extension workers in the north-west zone of Nigeria. International Journal of Agricultural Policy and Research 1(9):255-269. 